\title{
Dynamical inverse problem for the discrete Schrödinger operator
}

\author{
A. S. Mikhaylov ${ }^{1,2}$, V.S. Mikhaylov ${ }^{1,2}$ \\ ${ }^{1}$ St. Petersburg Department of V. A. Steklov Institute of Mathematics of the Russian Academy of Sciences, \\ 7, Fontanka, 191023, St. Petersburg, Russia \\ ${ }^{2}$ St. Petersburg State University, 7/9 Universitetskaya nab., 199034, St. Petersburg, Russia \\ a.mikhaylov@spbu.ru,v.mikhaylov@spbu.ru
}

\section{DOI 10.17586/2220-8054-2016-7-5-842-853}

We consider the inverse problem for the dynamical system with discrete Schrödinger operator and discrete time. As inverse data, we take a response operator, the natural analog of the dynamical Dirichlet-to-Neumann map. We derive two types of equations of inverse problem and answer a question on the characterization of the inverse data, i.e. we describe the set of operators, which are response operators of the dynamical system governed by the discrete Schrödinger operator.

Keywords: inverse problem, discrete Schrödinger operator, Boundary Control method, characterization of inverse data.

Received: 19 July 2016

Revised: 21 August 2016

Dedicated to the memory of B.S. Pavlov

\section{Introduction}

The theory of dynamical inverse problems is a wide area of modern mathematics, by now for all or almost all linear nonstationary equations of mathematical physics there exist an inverse theory more or less developed. Theories mostly covers the case of continuous problems, at the same time just a few attention is paid to discrete ones. The primary goal of the paper is to improve this situation.

Let $\mathbb{N}$ be the set of positive natural numbers, $\mathbb{N}_{0}=\mathbb{N} \cup\{0\}$. We fix the infinite sequence of real numbers $\left(b_{1}, b_{2}, \ldots\right)$, which we call the potential and consider the dynamical system with discrete time which is a natural analog of dynamical systems governed by the wave equation with potential on a semi-axis:

$$
\left\{\begin{array}{l}
u_{n, t+1}+u_{n, t-1}-u_{n+1, t}-u_{n-1, t}+b_{n} u_{n, t}=0, \quad n, t \in \mathbb{N}_{0} \\
u_{n,-1}=u_{n, 0}=0, \quad n \in \mathbb{N} \\
u_{0, t}=f_{t}, \quad t \in \mathbb{N}_{0}
\end{array}\right.
$$

By analogy with continuous problems [1], we treat the real sequence $f=\left(f_{0}, f_{1}, \ldots\right)$ as a boundary control. The solution to (1.1) we denote by $u_{n, t}^{f}$. to $u_{1, t}^{f}$ :

Having fixed $\tau \in \mathbb{N}$, with (1.1) we associate the response operators, which maps the control $f=\left(f_{0}, \ldots f_{\tau-1}\right)$

$$
\left(R^{\tau} f\right)_{t}:=u_{1, t}^{f}, \quad t=1, \ldots, \tau .
$$

The inverse problem we will be dealing with is to recover from $R^{\tau}$ (part of the) potential $\left(b_{1}, b_{2}, \ldots, b_{n}\right)$ for some $n$. This problems is a natural discrete analog of the inverse problem for the wave equation where the inverse data is the dynamical Dirichlet-to-Neumann map, see [1].

We will be using the Boundary Control method [1] which was initially developed to treat multidimensional dynamical inverse problems, but since then was applied to multi- and one- dimensional inverse dynamical, spectral and scattering problems, problems of signal processing and identification problems $[2,3]$.

In the second section, we study the forward problem: for (1.1) we prove the analog of d'Alembert integral representation formula. Prescribing the Dirichlet condition at $n=N+1$, we consider the second dynamical system with boundary control at $n=0$ (which will be an analog of the problem on the finite interval) and develop the solution of this system in Fourier series. We analyze the dependence of two solutions on the potential, which lead us to the natural set up of the inverse problem. In the third section, we introduce and prove the representation formulae for the main operators of the $\mathrm{BC}$ method: response operator, control and connecting operators. In the fourth section, we derive two types of equations for the inverse problem and give a characterization of the inverse data. In the last section, we highlight the connections between the different types of inverse data.

The case of the Jacobi matrices of general type as well as the studying of the inverse spectral problem, i.e. recovering the semi-infinite matrix from the spectral measure, will be the subject of forthcoming publications. 


\section{Forward problems}

We fix some positive integer $T$. By $\mathcal{F}^{T}$ we denote the space of controls: $\mathcal{F}^{T}:=\mathbb{R}^{T}, f \in \mathcal{F}^{T}, f=\left(f_{0}, \ldots, f_{T-1}\right)$.

First, we derive the representation formulas for the solution to (1.1) which could be considered as analogs of known formulas for the wave equation [4].

Lemma 1. The solution to (1.1) admits the representation:

$$
u_{n, t}=f_{t-n}+\sum_{s=n}^{t-1} w_{n, s} f_{t-s-1}, \quad n, t \in \mathbb{N}_{0}
$$

where $w_{n, s}$ satisfies the Goursat problem:

$$
\left\{\begin{array}{l}
w_{n, t+1}+w_{n, t-1}-w_{n+1, t}-w_{n-1, t}+b_{n} w_{n, t}=0, \quad n, s \in \mathbb{N}_{0}, s>n \\
w_{n, n}=-\sum_{k=1}^{n} b_{k}, \quad n \in \mathbb{N} \\
w_{0, t}=0, \quad t \in \mathbb{N}_{0} .
\end{array}\right.
$$

Proof. We assume that $u_{n, t}^{f}$ has a form (2.1) with unknown $w_{n, s}$ and plug it to equation in (1.1):

$$
0=b_{n} f_{t-n}+\sum_{s=n}^{t-1} b_{n} w_{n, s} f_{t-s-1}+\sum_{s=n}^{t} w_{n, s} f_{t-s}+\sum_{s=n}^{t-2} w_{n, s} f_{t-s-2}-\sum_{s=n+1}^{t-1} w_{n, s} f_{t-s-1}-\sum_{s=n-1}^{t-1} w_{n-1, s} f_{t-s-1} .
$$

Changing the order of summation, we get:

$$
\begin{aligned}
0 & =\quad b_{n} f_{t-n}+w_{n+1, n} f_{t-n-1}-w_{n-1, n-1} f_{t-n}+\sum_{s=n}^{t-1} f_{t-s-1}\left(b_{n} w_{n, s}-w_{n+1, s}-w_{n-1, s}\right) \\
& +\sum_{s=n-1}^{t-1} w_{n, s+1} f_{t-s-1}+\sum_{s=n+1}^{t-1} w_{n, s-1} f_{t-s-1}=f_{t-n-1}\left(w_{n+1, n}-w_{n, n-1}\right)+b_{n} f_{t-n} \\
& +\sum_{s=n}^{t-1} f_{t-s-1}\left(w_{n, s+1}+w_{n . s-1}-w_{n+1, s}-w_{n-1, s}+b_{n} w_{n, s}\right)+f_{t-n}\left(w_{n, n}-w_{n-1, n-1}\right) .
\end{aligned}
$$

Counting that $w_{n, s}=0$ when $n>s$ and arbitrariness of $f \in \mathcal{F}^{T}$, we arrive at (2.1).

We fix $N \in \mathbb{N}$. Along with (1.1) we consider the analog of the wave equation with the potential on the interval: we assume that $\left(b_{n}\right)$ is finite: $n=1, \ldots, N$ and impose the Dirichlet condition at $n=N+1$. Then for a control $f=\left(f_{0}, f_{1}, \ldots\right)$ we consider:

$$
\left\{\begin{array}{l}
v_{n, t+1}+v_{n, t-1}-v_{n+1, t}-v_{n-1, t}+b_{n} v_{n, t}=0, \quad t \in \mathbb{N}_{0}, n \in 0, \ldots, N+1 \\
v_{n,-1}=v_{n, 0}=0, \quad n=1,2, \ldots, N+1 \\
v_{0, t}=f_{t}, \quad v_{N+1, t}=0, \quad t \in \mathbb{N}_{0} .
\end{array}\right.
$$

We denote the solution to (2.3) by $v^{f}$.

Let $\phi_{n}(\lambda)$ be the solution to:

$$
\left\{\begin{array}{l}
\phi_{i+1}+\phi_{i-1}-b_{n} \phi_{i}=\lambda \phi_{i} \\
\phi_{0}=0, \phi_{1}=1
\end{array}\right.
$$

We introduce the Hamiltonian:

$$
H_{N}:=\left(\begin{array}{ccccc}
-b_{1} & 1 & 0 & \ldots & 0 \\
1 & -b_{2} & 1 & \ldots & 0 \\
\cdot & \cdot & \cdot & \cdot & \cdot \\
0 & \ldots & 0 & 1 & -b_{N}
\end{array}\right)
$$

Let $\left\{\varphi^{k}, \lambda_{k}\right\}_{k=1}^{N}$ be eigenvectors chosen such that $\varphi_{1}^{k}=1$ and eigenvalues of $H_{N}$. Introduce the numbers $\rho_{k}$ by:

$$
\left(\varphi^{k}, \varphi^{l}\right)=\delta_{k l} \rho_{k}
$$

where $(\cdot, \cdot)$ - is a scalar product in $\mathbb{R}^{N}$. 
Definition 1. The set

is called the spectral data.

$$
\left\{\lambda_{k}, \rho_{k}\right\}_{k=1}^{N}
$$

On introducing vectors $\phi^{n} \in \mathbb{R}^{N}$ by the rule $\phi_{i}^{n}:=\phi_{i}\left(\lambda_{n}\right), n, i=1, \ldots, N$, we have

Proposition 1. The solutions of $\phi_{N+1}(\lambda)=0$ are $\lambda_{n}, n=1, \ldots, N$; and $\phi_{i}^{n}=\varphi_{i}^{n}, n, i=1, \ldots, N$.

Proof. Take eigenvector $\varphi^{n}$ corresponding to eigenvalue $\lambda_{n}$ and compare it with $\phi^{n}$. By the definition of $\varphi^{n}$ and condition in (2.4): $\varphi_{1}^{n}=\phi_{1}^{n}=1$. On the other hand, comparing the first line in the equation on eigenvalues $H_{N} \varphi^{n}=\lambda^{n} \varphi^{n}$ and (2.4) for $i=1$ we have:

$$
\begin{array}{r}
-\varphi_{1}^{n} b_{1}+\varphi_{2}^{n}=\lambda_{n} \varphi_{1}^{n}, \\
\phi_{2}^{n}-b_{1} \phi_{1}^{n}=\lambda_{n} \phi_{1}^{n},
\end{array}
$$

which implies $\varphi_{2}^{n}=\phi_{2}^{n}$, for $k<N$ comparing $k$-th line in $H_{N} \varphi^{n}=\lambda^{n} \varphi^{n}$ and (2.4) for $i=k$, we arrive at $\varphi_{k+1}^{n}=\phi_{k+1}^{n}$. And for $k=N$ :

$$
\begin{array}{r}
-\varphi_{N}^{n} b_{N}+\varphi_{N-1}^{n}=\lambda_{n} \varphi_{N}^{n}, \\
\phi_{N+1}^{n}+\phi_{N-1}^{n}-b_{N} \phi_{N}^{n}=\lambda_{n} \phi_{N}^{n},
\end{array}
$$

which holds if and only if $\phi_{N+1}^{n}\left(\lambda_{n}\right)=0$.

We take $y \in \mathbb{R}^{N}, y=\left(y_{1}, \ldots, y_{N}\right)$, for each $n$ we multiply the equation in (2.3) by $y_{n}$, sum up and evaluate the following expression, changing the order of summation:

$$
\begin{array}{r}
0=\sum_{n=1}^{N}\left(v_{n, t+1} y_{n}+v_{n, t-1} y_{n}-v_{n+1, t} y_{n}-v_{n-1, t} y_{n}+b_{n} v_{n, t} y_{n}\right)= \\
\sum_{n=1}^{N}\left(v_{n, t+1} y_{n}+v_{n, t-1} y_{n}-v_{n, t}\left(y_{n-1}+y_{n+1}\right)+b_{n} v_{n, t} y_{n}\right)-v_{N+1, t} y_{N}-v_{0, t} y_{1}+v_{1, t} y_{0}+v_{N, t} y_{N+1} .
\end{array}
$$

Now, we choose $y=\varphi^{l}, l=1 \ldots, N$. On counting that $\varphi_{0}^{l}=\varphi_{N+1}^{l}=0, \varphi_{1}^{l}=1, v_{0, t}=f_{t}, v_{N+1, t}=0$ we evaluate (2.7) arriving at:

$$
0=\sum_{n=1}^{N}\left(v_{n, t+1} \varphi_{n}^{l}+v_{n, t-1} \varphi_{n}^{l}-v_{n, t}\left(\varphi_{n-1}^{l}+\varphi_{n+1}^{l}-b_{n} \varphi_{n}^{l}\right)\right)-f_{t}=0 .
$$

Definition 2. For $a, b \in l^{\infty}$, we define the convolution $c=a * b \in l^{\infty}$ by the formula:

$$
c_{t}=\sum_{s=0}^{t} a_{s} b_{t-s}, \quad t \in \mathbb{N} .
$$

We assume that the solution to (2.3) has the form:

$$
v_{n, t}^{f}=\left\{\begin{array}{l}
\sum_{k=1}^{N} c_{t}^{k} \varphi_{n}^{k}, \quad n=1, \ldots, N \\
f_{t}, \quad n=0 .
\end{array}\right.
$$

Proposition 2. The coefficients $c^{k}$ admits the representation:

$$
c^{k}=\frac{1}{\rho_{k}} T\left(\lambda_{k}\right) * f
$$

where $T(2 \lambda)=\left(T_{1}(2 \lambda), T_{2}(2 \lambda), T_{3}(2 \lambda), \ldots\right)$ are Chebyshev polynomials of the second kind.

Proof. We plug (2.9) into (2.8) and evaluate, counting that $\varphi_{n-1}^{l}+\varphi_{n+1}^{l}-b_{n} \varphi_{n}^{l}=\lambda_{l} \varphi_{n}^{l}$ :

$$
\begin{aligned}
\sum_{n=1}^{N}\left(v_{n, t+1}+v_{n, t-1}-\lambda_{l} v_{n, t}\right) \varphi_{n}^{l} & =f_{t}, \\
\sum_{n=1}^{N} \sum_{k=1}^{N}\left(c_{t+1}^{k} \varphi_{n}^{k}+c_{t-1}^{k} \varphi_{n}^{k}-\lambda_{l} c_{t}^{k} \varphi_{n}^{k}\right) \varphi_{n}^{l} & =f_{t} .
\end{aligned}
$$


Changing the order of summation and using (2.5), we finally arrive at the following equation on $c_{t}^{k}, k=1, \ldots, N$ :

$$
\left\{\begin{array}{l}
c_{t+1}^{k}+c_{t-1}^{k}-\lambda_{k} c_{t}^{k}=\frac{1}{\rho_{k}} f_{t}, \\
c_{-1}^{k}=c_{0}^{k}=0
\end{array}\right.
$$

We assume that the solution to (2.11) has the form: $c^{k}=\frac{1}{\rho_{k}} T * f$, or

$$
c_{t}^{k}=\frac{1}{\rho_{k}} \sum_{l=0}^{t} T_{l} f_{t-l}
$$

Plugging it into (2.11), we get:

$$
\begin{gathered}
\frac{1}{\rho_{k}}\left(\sum_{l=0}^{t+1} f_{l} T_{t+1-l}+\sum_{l=0}^{t-1} f_{l} T_{t-1-l}-\lambda_{k} \sum_{l=0}^{t} f_{l} T_{t-l}\right)=\frac{1}{\rho_{k}} f_{t}, \\
\sum_{l=0}^{t} f_{l}\left(T_{t+1-l}+T_{t-1-l}-\lambda_{k} T_{t-l}\right)+f_{t} T_{1}-f_{t-1} T_{0}=f_{t} .
\end{gathered}
$$

We see that (2.12) holds if $T$ solves:

$$
\left\{\begin{array}{l}
T_{t+1}+T_{t-1}-\lambda_{k} T_{t}=0 \\
T_{0}=0, T_{1}=1
\end{array}\right.
$$

Thus $T_{k}(2 \lambda)$ are Chebyshev polynomials of the second kind.

\section{Operators of the the BC method}

As inverse data for (1.1), we use the analog of the dynamical response operator (dynamical Dirichlet-toNeumann map) [1].

Definition 3. For (1.1), the response operator $R^{T}: \mathcal{F}^{T} \mapsto \mathbb{R}^{T}$ is defined by the rule

$$
\left(R^{T} f\right)_{t}=u_{1, t}^{f}, \quad t=1, \ldots, T \text {. }
$$

Introduce the notation: the response vector is the convolution kernel of the response operator, $r=\left(r_{0}, r_{1}, \ldots, r_{T-1}\right)=$ $\left(1, w_{1,1}, w_{1,2}, \ldots w_{1, T-1}\right)$. Then, in accordance with (2.1):

$$
\begin{array}{r}
\left(R^{T} f\right)_{t}=u_{1, t}^{f}=f_{t-1}+\sum_{s=1}^{t-1} w_{1, s} f_{t-1-s}, \quad t=1, \ldots, T \\
\left(R^{T} f\right)=r * f_{--1}, \quad \text { where } r_{0}=1 .
\end{array}
$$

For system (2.3), we introduce the response operator by:

Definition 4. For the system in (2.3) the response operator $R_{i}^{T}: \mathcal{F}^{T} \mapsto \mathbb{R}^{T}$ is defined by the rule:

$$
\left(R_{i}^{T} f\right)_{t}=v_{1, t}^{f}, \quad t=1, \ldots, T .
$$

The corresponding response vector we denote by $\left(r_{1}^{i}, r_{2}^{i}, \ldots\right)$. More information on this operator and on the inverse spectral problem one can find in the last section.

We introduce the inner space of dynamical system (1.1) $\mathcal{H}^{T}:=\mathbb{R}^{T}, h \in H^{T}, h=\left(h_{1}, \ldots, h_{T}\right)$. For (1.1) The control operator $W^{T}: \mathcal{F}^{T} \mapsto \mathcal{H}^{T}$ is defined by the rule:

$$
W^{T} f:=u_{n, T}^{f}, \quad n=1, \ldots, T .
$$

Directly from (2.1), we deduce that:

$$
\left(W^{T} f\right)_{n}=u_{n, T}^{f}=f_{T-n}+\sum_{s=n}^{T-1} w_{n, s} f_{T-s-1}, \quad n=1, \ldots, T .
$$

The following statement imply the controllability of the dynamical system (1.1). 
Theorem 1. The operator $W^{T}$ is an isomorphism between $\mathcal{F}^{T}$ and $\mathcal{H}^{T}$.

Proof. We fix some $a \in \mathcal{H}^{T}$ and look for a control $f \in \mathcal{F}^{T}$ such that $W^{T} f=a$. To this aim we write down the operator as:

$$
W^{T} f=\left(\begin{array}{c}
u_{1, T} \\
u_{2, T} \\
\cdot \\
u_{k, T} \\
\cdot \\
u_{T, T}
\end{array}\right)=\left(\begin{array}{cccccc}
1 & w_{1,1} & w_{1,2} & \ldots & \ldots & w_{1, T-1} \\
0 & 1 & w_{2,2} & \ldots & \ldots & w_{2, T-1} \\
\cdot & \cdot & \cdot & \cdot & \cdot & \cdot \\
0 & \ldots & 1 & w_{k, k} & \ldots & w_{k, T-1} \\
\cdot & \cdot & \cdot & \cdot & \cdot & \cdot \\
0 & 0 & 0 & 0 & \ldots & 1
\end{array}\right)\left(\begin{array}{c}
f_{T-1} \\
f_{T-2} \\
\cdot \\
f_{T-k-1} \\
\cdot \\
f_{0}
\end{array}\right)
$$

We introduce the notations:

$$
\begin{array}{r}
J_{T}: \mathcal{F}^{T} \mapsto \mathcal{F}^{T}, \quad\left(J_{T} f\right)_{n}=f_{T-1-n}, \quad n=0, \ldots, T-1, \\
K \in \mathbb{R}^{T \times T}, \quad k_{i j}=0, \quad i>j, \quad k_{i i}=1, \quad k_{i j}=w_{i j-1}, \quad i<j .
\end{array}
$$

Then, $W^{T}=(I+K) J^{T}$. Obviously, this operator is invertible, which proves the statement of the theorem.

For the system (2.3) the control operator $W_{i}^{T}: \mathcal{F}^{T} \mapsto \mathcal{H}^{N}$ is defined by the rule:

$$
W_{i}^{T} f:=v_{n, T}^{f}, \quad n=1, \ldots, N .
$$

The representation for this operator immediately follows from (2.9), (2.10).

For the system (1.1) we introduce the connecting operator $C^{T}: \mathcal{F}^{T} \mapsto \mathcal{F}^{T}$ by the quadratic form: for arbitrary $f, g \in \mathcal{F}^{T}$ we define

$$
\left(C^{T} f, g\right)_{\mathcal{F}^{T}}=\left(u_{\cdot, T}^{f}, u_{\cdot, T}^{g}\right)_{\mathcal{H}^{T}}=\left(W^{T} f, W^{T} g\right)_{\mathcal{H}^{T}} .
$$

We observe that $C^{T}=\left(W^{T}\right)^{*} W^{T}$, so $C^{T}$ is an isomorphism in $\mathcal{F}^{T}$. The fact that $C^{T}$ can be expressed in terms of response $R^{2 T}$ is crucial in BC-method.

Theorem 2. Connecting operator admits the representation in terms of inverse data:

$$
\begin{aligned}
& C^{T}=C_{i j}^{T}, \quad C_{i j}^{T}=\sum_{k=0}^{T-\max i, j} r_{|i-j|+2 k}, \quad r_{0}=1 . \\
& C^{T}=\left(\begin{array}{ccccc}
1+r_{2}+\ldots+r_{2 T-2} & r_{1}+r_{3}+\ldots+r_{2 T-3} & \ldots & r_{T}+r_{T-2} & r_{T-1} \\
r_{1}+r_{3}+\ldots+r_{2 T-3} & 1+r_{2}+\ldots+r_{2 T-4} & \ldots & \ldots & r_{T-2} \\
\cdot & . & . & . & \cdot \\
r_{T-3}+r_{T-1}+r_{T+1} & \ldots & 1+r_{2}+r_{4} & r_{1}+r_{3} & r_{2} \\
r_{T}+r_{T-2} & \ldots & r_{1}+r_{3} & 1+r_{2} & r_{1} \\
r_{T-1} & r_{T-2} & \ldots & r_{1} & 1
\end{array}\right)
\end{aligned}
$$

Proof. For fixed $f, g \in \mathcal{F}^{T}$, we introduce the Blagoveshchensky function by:

$$
\psi_{n, t}:=\left(u_{\cdot, n}^{f}, u_{\cdot, t}^{g}\right)_{\mathcal{H}^{T}}=\sum_{k=1}^{T} u_{k, n}^{f} u_{k, t}^{g} .
$$

Then, we show that $\psi_{n, t}$ satisfies some difference equation. Indeed, we can evaluate:

$$
\begin{gathered}
\psi_{n, t+1}+\psi_{n, t-1}-\psi_{n+1, t}-\psi_{n-1, t}= \\
\sum_{k=1}^{T} u_{k, n}^{f}\left(u_{k, t+1}^{g}+u_{k, t-1}^{g}\right)-\sum_{k=1}^{T}\left(u_{k, n+1}^{f}+u_{k, n-1}^{f}\right) u_{k, t}^{g}= \\
\sum_{k=1}^{T} u_{k, n}^{f}\left(u_{k+1, t}^{g}+u_{k-1, t}^{g}\right)-\sum_{k=1}^{T}\left(u_{k+1, n}^{f}+u_{k-1, n}^{f}\right) u_{k, t}^{g}= \\
\sum_{k=1}^{T} u_{k, t}^{g}\left(u_{k+1, n}^{f}+u_{k-1, n}^{f}\right)+u_{0, t}^{g} u_{1, n}^{f}-u_{0, n}^{f} u_{1, t}^{g}+u_{T+1, t}^{g} u_{T, n}^{f}-u_{T+1, n}^{f} u_{T, t}^{g}-\sum_{k=1}^{T} u_{k, t}^{g}\left(u_{k+1, n}^{f}+u_{k-1, n}^{f}\right)= \\
g_{t}(R f)_{n}-f_{n}(R g)_{t} .
\end{gathered}
$$


So, we arrive at the following boundary problem for $\psi_{n, t}$ :

$$
\left\{\begin{array}{l}
\psi_{n, t+1}+\psi_{n, t-1}-\psi_{n+1, t}-\psi_{n-1, t}=h_{n, t}, \quad n, t \in \mathbb{N}_{0} \\
\psi_{0, t}=0, \psi_{n, 0}=0 \\
h_{n, t}=g_{t}(R f)_{n}-f_{n}(R g)_{t} .
\end{array}\right.
$$

We introduce the set:

$$
\begin{aligned}
K(n, t):= & \{(n, t) \cup\{(n-1, t-1),(n+1, t-1)\} \cup\{(n-2, t-2),(n, t-2),(n+2, t-2)\} \cup \ldots \\
& \cup\{(n-t, 0),(n-t+2,0), \ldots,(n+t-2,0),(n+t, 0)\}\}=\bigcup_{\tau=0}^{t} \bigcup_{k=0}^{\tau}(n-\tau+2 k, t-\tau) .
\end{aligned}
$$

The solution to (3.7) is given by:

$$
\psi_{n, t}=\sum_{k, \tau \in K(n, t-1)} h(k, \tau)
$$

We observe that $\psi_{T, T}=\left(C^{T} f, g\right)$, so:

$$
\left(C^{T} f, g\right)=\sum_{k, \tau \in K(T, T-1)} h(k, \tau) .
$$

Notice that in the r.h.s. of (3.8) the argument $k$ runs from 1 to $2 T-1$. We extend $f \in \mathcal{F}^{T}, f=\left(f_{0}, \ldots, f_{T-1}\right)$ to $f \in \mathcal{F}^{2 T}$ by:

\[ f_{T}=0, \quad f_{T+k}=-f_{T-k}, \quad k=1,2, \ldots \]
$f_{k}\left(R^{T} g\right)_{\tau}=0$, so (3.8) gives:

$$
\begin{aligned}
\left(C^{T} f, g\right)=\sum_{k, \tau \in K(T, T-1)} g_{\tau}\left(R^{2 T} f\right)_{k} & =g_{0}\left[\left(R^{2 T} f\right)_{1}+\left(R^{2 T} f\right)_{3}+\ldots+\left(R^{2 T} f\right)_{2 T-1}\right] \\
& +g_{1}\left[\left(R^{2 T} f\right)_{2}+\left(R^{2 T} f\right)_{4}+\ldots+\left(R^{2 T} f\right)_{2 T-2}\right]+\ldots+g_{T-1}\left(R^{2 T} f\right)_{T}
\end{aligned}
$$

Finally, we infer that:

$$
C^{T} f=\left(\left(R^{2 T} f\right)_{1}+\ldots+\left(R^{2 T} f\right)_{2 T-1},\left(R^{2 T} f\right)_{2}+\ldots+\left(R^{2 T} f\right)_{2 T-2}, \ldots,\left(R^{2 T} f\right)_{T}\right)
$$

from where the statement of the theorem follows.

One can observe that $C_{i j}^{T}$ satisfies the difference boundary problem.

Corollary 1. The kernel of $C^{T}$ satisfy:

$$
\left\{\begin{array}{l}
C_{i, j+1}^{T}+C_{i, j-1}^{T}-C_{i+1, j}^{T}-C_{i-1, j}^{T}=0 \\
C_{i, T}^{T}=r_{T-i}, C_{T, j}^{T}=r_{T-j}, r_{0}=1
\end{array}\right.
$$

For the system (2.3) the connecting operator $C_{i}^{T}: \mathcal{F}^{T} \mapsto \mathcal{F}^{T}$ is introduced in the similar way: for arbitrary $f, g \in \mathcal{F}^{T}$ we define:

$$
\left(C_{i}^{T} f, g\right)_{\mathcal{F}^{T}}=\left(v_{\cdot, T}^{f}, v_{\cdot, T}^{g}\right)_{\mathcal{H}^{N}}=\left(W_{i}^{T} f, W_{i}^{T} g\right)_{\mathcal{H}^{N}} .
$$

More information on $C_{i}^{T}$ one can find in the final section.

\section{Inverse problem}

The dependence of the solution (1.1) $u^{f}$ on the potential $\left(b_{1}, b_{2}, \ldots\right)$ resembles one of the wave equation with the potential: take some $M \in \mathbb{N}$. From the very equation, one can see that the term $u_{n, t}^{f}$ with smallest $\{n, t\}$, which depends on $b_{M}$ is $u_{M, M+1}^{f}$. Thus, $u_{1, t}^{f}$ becomes dependent upon $b_{M}$ starting from $t=2 M$. This is an analog of the finite wave propagation speed effect in the wave equation. Consider (2.3) with $N=M$. We see that the solution to (2.3) $v_{1, t}^{f}$ does not 'feel' the boundary condition at $n=M+1: u_{1, t}^{f}=v_{1, t}^{f}$ for $t=1, \ldots, 2 M$. Or in other words, that means that $R^{2 M}=R_{i}^{2 M}$. This leads to the following natural set up of the inverse problem: By the given operator $R^{2 M}$ to recover the (part) of the potential $\left(b_{1}, \ldots, b_{M}\right)$. In what follows, we will be dealing with the IP for the system (1.1), only in the last section we comment on the system (2.3). 


\subsection{Krein equations}

Let $\alpha, \beta \in \mathbb{R}$ and $y$ be solution to:

$$
\left\{\begin{array}{l}
y_{k+1}+y_{k-1}-b_{k} y_{k}=0 \\
y_{0}=\alpha, y_{1}=\beta
\end{array}\right.
$$

We set up the following control problem: to find a control $f^{T} \in \mathcal{F}^{T}$ such that:

$$
\left(W^{T} f^{T}\right)_{k}=y_{k}, \quad k=1, \ldots, T .
$$

Due to Theorem 1, this problem has unique solution. Let $\varkappa^{T}$ be a solution to

$$
\left\{\begin{array}{l}
\varkappa_{t+1}^{T}+\varkappa_{t-1}^{T}=0, \quad t=0, \ldots, T \\
\varkappa_{T}^{T}=0, \varkappa_{T-1}^{T}=1
\end{array}\right.
$$

We show that the control $f^{T}$ satisfies the Krein equation:

Theorem 3. The control $f^{T}$, defined by (4.2) satisfies the following equation in $\mathcal{F}^{T}$ :

$$
C^{T} f^{T}=\beta \varkappa^{T}-\alpha\left(R^{T}\right)^{*} \varkappa^{T}
$$

Proof. Let us take $f^{T}$ solving (4.2). We observe that for any fixed $g \in \mathcal{F}^{T}$ :

$$
u_{k, T}^{g}=\sum_{t=1}^{T-1}\left(u_{k, t+1}^{g}+u_{k, t-1}^{g}\right) \varkappa_{t}^{T} .
$$

Indeed, changing the order of summation in the r.h.s. of (4.5), we get:

$$
\sum_{t=1}^{T-1}\left(u_{k, t+1}^{g}+u_{k, t-1}^{g}\right) \varkappa_{t}^{T}=\sum_{t=1}^{T-1}\left(\varkappa_{t+1}^{T}+\varkappa_{t-1}^{T}\right) u_{k, t}^{g}+u_{k, 0}^{g} \varkappa_{1}^{T}-u_{k, T}^{g} \varkappa_{T-1}^{T} .
$$

which gives (4.5) due to (4.3). Using this observation, we can evaluate:

$$
\begin{gathered}
\left(C^{T} f^{T}, g\right)=\sum_{k=1}^{T} y_{k} u_{k, T}^{g}=\sum_{k=1}^{T} \sum_{t=0}^{T-1}\left(u_{k, t+1}^{g}+u_{k, t-1}^{g}\right) \varkappa_{t}^{T} y_{k} \\
=\sum_{t=0}^{T-1} \varkappa_{t}^{T}\left(\sum_{k=1}^{T}\left(u_{k+1, t}^{g} y_{k}+u_{k-1, t}^{g} y_{k}-b_{k} u_{k, t}^{g} y_{k}\right)\right) \\
=\sum_{t=0}^{T-1} \varkappa_{t}^{T}\left(\sum_{k=1}^{T}\left(u_{k, t}^{g}\left(y_{k+1}+y_{k-1}-b_{k} y_{k}\right)+u_{0, t}^{g} y_{1}+u_{T+1, t}^{g} y_{T}-u_{1, t}^{g} y_{0}-u_{T, t}^{g} y_{T+1}\right)\right. \\
=\sum_{t=0}^{T-1} \varkappa_{t}^{T}\left(\beta g_{t}-\alpha\left(R^{T} g\right)_{t}\right)=\left(\varkappa^{T}, \beta g-\alpha\left(R^{T} g\right)\right)=\left(\beta \varkappa^{T}-\alpha\left(\left(R^{T}\right)^{*} \varkappa^{T}\right), g\right) .
\end{gathered}
$$

From where (4.4) follows.

Having found $f^{\tau}$ for $\tau=1, \ldots, T$, we can recover the potential $b_{n}, n=1, \ldots, T-1$. Indeed: by the constructions of $f^{\tau}$ we have $\left(W^{\tau} f^{\tau}\right)_{\tau}=y_{\tau}$, on the other hand, from (3.3) we can infer that $\left(W^{\tau} f^{\tau}\right)_{\tau}=f_{0}^{\tau}$, thus $y$ (4.1) can be recovered by:

$$
y_{\tau}=f_{0}^{\tau}, \quad \tau=1, \ldots, T
$$

And the potential can be found by:

$$
b_{n}=\frac{y_{n+1}+y_{n-1}}{y_{n}}, \quad n=1, \ldots, T-1 .
$$




\subsection{Factorization method}

We make use the fact that matrix $C^{T}$ has a special structure - it is a product of triangular matrix and its conjugate. We rewrite the operator $W^{T}=\bar{W}^{T} J$ as:

$$
W^{T} f=\left(\begin{array}{ccccc}
1 & w_{1,1} & w_{1,2} & \ldots & w_{1, T-1} \\
0 & 1 & w_{2,2} & \ldots & w_{2, T-1} \\
\cdot & \cdot & \cdot & \cdot & \cdot \\
0 & \ldots & 1 & \ldots & w_{k, T-1} \\
\cdot & \cdot & \cdot & . & \cdot \\
0 & 0 & 0 & \ldots & 1
\end{array}\right)\left(\begin{array}{ccccc}
0 & 0 & 0 & \ldots & 1 \\
0 & 0 & 0 & \ldots & 0 \\
\cdot & \cdot & \cdot & \cdot & \cdot \\
0 & \ldots & 1 & 0 & 0 \\
\cdot & \cdot & \cdot & \cdot & \cdot \\
1 & 0 & 0 & 0 & 0
\end{array}\right)\left(\begin{array}{c}
f_{0} \\
f_{2} \\
\cdot \\
f_{T-k-1} \\
\cdot \\
f_{T-1}
\end{array}\right)
$$

Using the definition (3.5) and the invertibility of $W^{T}$ (cf. Theorem 1), we have:

$$
C^{T}=\left(W^{T}\right)^{*} W^{T}, \quad \text { or } \quad\left(\left(W^{T}\right)^{-1}\right)^{*} C^{T}\left(W^{T}\right)^{-1}=I .
$$

We can rewrite the latter equation as:

$$
\left(\left(\bar{W}^{T}\right)^{-1}\right)^{*} \bar{C}^{T}\left(\bar{W}^{T}\right)^{-1}=I, \quad \bar{C}^{T}=J C^{T} J
$$

Here the matrix $\bar{C}^{T}$ has the entries:

$$
\bar{C}_{i j}=C_{T+1-j, T+1-i}, \quad \bar{C}^{T}=\left(\begin{array}{ccccc}
1 & r_{1} & r_{2} & \ldots & r_{T-1} \\
r_{1} & 1+r_{2} & r_{1}+r_{3} & \ldots & . . \\
r_{3} & r_{1}+r_{3} & 1+r_{2}+r_{4} & \ldots & . . \\
\cdot & \cdot & . & . & .
\end{array}\right),
$$

and operator $\left(\bar{W}^{T}\right)^{-1}$ has the form:

$$
\left(\bar{W}^{T}\right)^{-1}=\left(\begin{array}{ccccc}
1 & \widetilde{k}_{11} & \widetilde{k}_{12} & \ldots & \widetilde{k}_{1, T-1} \\
0 & 1 & \widetilde{k}_{22} & \cdots & \ldots \\
\cdot & \cdot & \cdot & \cdot & \widetilde{k}_{T-1, T-1} \\
0 & \ldots & \ldots & 0 & 1
\end{array}\right)
$$

where $\widetilde{k}_{\alpha, \alpha}=-w_{\alpha, \alpha}, \alpha=1, \ldots, T-1$. So we can rewrite (4.8) as:

$$
\left(\begin{array}{cccc}
1 & 0 & . & 0 \\
k_{11} & 1 & 0 & . \\
. & . & . & . \\
k_{T-1,1} & . & . & 1
\end{array}\right)\left(\begin{array}{cccc}
\bar{c}_{11} & . . & . . & \bar{c}_{1 T} \\
. . & . . & . . & . . \\
. & . & . & . \\
\bar{c}_{T 1} & . . & & \bar{c}_{T T}
\end{array}\right)\left(\begin{array}{cccc}
1 & k_{11} & k_{21} & . . \\
0 & 1 & k_{22} & . . \\
. & . & . & . \\
0 & . . & . . & 1
\end{array}\right)=\left(\begin{array}{cccc}
1 & 0 & . . & 0 \\
0 & 1 & . . & 0 \\
. & . & . & . \\
0 & 0 & . & 1
\end{array}\right) .
$$

In the above equation $\bar{C}_{i j}$ are given (see (4.9)), the entries $k_{i j}$ of $\left(\left(\bar{W}^{T}\right)^{-1}\right)^{*}$ are unknown. We denote by $K_{i}:=\left(k_{i 1}, k_{i 2}, \ldots, k_{i i}, 1,0, \ldots, 0\right)$ the $(i+1)$-th row $(i=0, \ldots, T-1)$ in $\left(\left(\bar{W}^{T}\right)^{-1}\right)^{*}$, then we have

$$
K_{i} \bar{C}^{T} K_{j}^{*}=\delta_{i, j}
$$

We use this equality in the form:

$$
K_{i} \bar{C}^{T} K_{j}^{*}=0, \quad i<j .
$$

Notice that $K_{0}=(1,0, \ldots, 0)$. The second row $K_{1}$ can be recovered from $K_{0} \bar{C}^{T} K_{1}^{*}=0$, which is equivalent to:

$$
\bar{c}_{11} k_{11}+\bar{c}_{21}=0, \text { or } k_{11}=-\frac{\bar{c}_{21}}{\bar{c}_{11}}=-\bar{c}_{21} .
$$

The third row $K_{2}$ we recover from the pair of equations $K_{0} \bar{C}^{T} K_{2}^{*}=0, K_{1} \bar{C}^{T} K_{2}^{*}=0$, which are equivalent to:

$$
\left(\begin{array}{cc}
1 & 0 \\
k_{11} & 1
\end{array}\right)\left(\begin{array}{lll}
\bar{c}_{11} & \bar{c}_{12} & \bar{c}_{13} \\
\bar{c}_{21} & \bar{c}_{22} & \bar{c}_{23}
\end{array}\right)\left(\begin{array}{c}
k_{21} \\
k_{22} \\
1
\end{array}\right)=\left(\begin{array}{l}
0 \\
0
\end{array}\right)
$$


Due to the invertibility of $\left(\begin{array}{cc}1 & 0 \\ k_{1,1} & 1\end{array}\right)$, we can rewrite the latter equation as:

$$
\left(\begin{array}{ll}
\bar{c}_{11} & \bar{c}_{12} \\
\bar{c}_{21} & \bar{c}_{22}
\end{array}\right)\left(\begin{array}{l}
k_{21} \\
k_{22}
\end{array}\right)=-\left(\begin{array}{l}
\bar{c}_{13} \\
\bar{c}_{23}
\end{array}\right) .
$$

We introduce the notation, by $c_{i}^{k}$ we denote the i-th column in the matrix $\bar{C}^{T}$ truncated by first $k$ elements:

$$
\bar{c}_{i}^{k}:=\left(\begin{array}{llll}
\bar{c}_{1 i} & \bar{c}_{2 i} & \ldots & \bar{c}_{k i}
\end{array}\right)^{*} .
$$

Since $C^{T}$ is invertible, (4.13) has a unique solution, moreover, we can infer that:

$$
k_{22}=-\frac{\operatorname{det}\left(\begin{array}{ll}
\bar{c}_{11} & \bar{c}_{13} \\
\bar{c}_{21} & \bar{c}_{23}
\end{array}\right)}{\operatorname{det}\left(\begin{array}{ll}
\bar{c}_{11} & \bar{c}_{12} \\
\bar{c}_{21} & \bar{c}_{22}
\end{array}\right)}=-\operatorname{det}\left(\bar{c}_{1}^{2}, \bar{c}_{3}^{2}\right)
$$

Assume that we have already recovered $K_{0}, K_{1}, \ldots, K_{l}$, to recover $K_{l+1}$ we need to consider the equations $K_{0} \bar{C}^{T} K_{l+1}^{*}=0, K_{1} \bar{C}^{T} K_{l+1}^{*}=0, \ldots, K_{l} \bar{C}^{T} K_{l+1}^{*}=0$, which are equivalent to:

$$
\left(\begin{array}{cccc}
1 & 0 & . . & 0 \\
k_{11} & 1 & 0 & . . \\
. & . & . & . \\
k_{l 1} & k_{l 2} & . & 1
\end{array}\right)\left(\begin{array}{cccc}
\bar{c}_{11} & . . & . . & \bar{c}_{1, l+2} \\
. . & . . & . . & . . \\
. . & . . & . . & . . \\
\bar{c}_{l+1,1} & . . & . . & \bar{c}_{l+1, l+2}
\end{array}\right)\left(\begin{array}{c}
k_{l+1,1} \\
k_{l+1,2} \\
. . \\
1
\end{array}\right)=\left(\begin{array}{c}
0 \\
. . \\
. . \\
0
\end{array}\right) .
$$

We can rewrite the latter equation as:

$$
\left(\begin{array}{cccc}
\bar{c}_{1,1} & . . & . . & \bar{c}_{1, l+1} \\
. . & . . & . . & . . \\
. . & . . & . . & . . \\
\bar{c}_{l+1,1} & . . & . . & \bar{c}_{l+1, l+1}
\end{array}\right)\left(\begin{array}{c}
k_{l+1,1} \\
k_{l+1,2} \\
. . \\
k_{l+1, l+1}
\end{array}\right)+\left(\begin{array}{c}
\bar{c}_{1, l+2} \\
\bar{c}_{2, l+2} \\
. . \\
\bar{c}_{l+1, l+2}
\end{array}\right)=0 .
$$

Due to the invertibility of $C^{T}$ the latter equation has unique solution, moreover

$$
k_{l+1, l+1}=-\operatorname{det}\left(\bar{c}_{1}^{l+1}, \bar{c}_{2}^{l+1}, \ldots \bar{c}_{l}^{l+1}, \bar{c}_{l+2}^{l+1}\right), \quad l=0, \ldots, T-2 .
$$

Having recovered $k_{\alpha, \alpha}=-w_{\alpha, \alpha}$, we recover the potential by (see (2.2)):

$$
b_{n}=w_{n-1, n-1}-w_{n, n}=k_{n, n}-k_{n-1, n-1}, \quad n=1, \ldots, T-1 .
$$

\subsection{Gelfand-Levitan equations}

If we introduce $\widetilde{C}^{T}$ by

$$
\bar{C}^{T}=I+\widetilde{C}^{T},
$$

(see (3.6),(4.9)), then we can rewrite (4.14) for $l=T-2$ as:

$$
\left(I+\widetilde{C}^{T}\right) K_{T}+\widetilde{C}_{T}=0, \text { where } K_{T}=\left(\begin{array}{c}
k_{T-1,1} \\
k_{T-1,2} \\
\cdot \\
k_{T-1, T-1}
\end{array}\right), \widetilde{C}_{T}=\left(\begin{array}{c}
\widetilde{C}_{1, T}^{T} \\
\widetilde{C}_{2, T}^{T} \\
\cdot \\
\widetilde{C}_{T-1, T}^{T}
\end{array}\right)
$$

or as a system:

$$
k_{T-1, \beta}+\sum_{j=1}^{T-1} \widetilde{C}_{\beta, j}^{T} k_{T-1, j}+\widetilde{C}_{\beta, T}^{T}=0, \quad \beta=1, \ldots, T-1 .
$$

If we pass to (more standard) entries of $\left(\bar{W}^{T}\right)^{-1}$

$$
\widetilde{k}_{\alpha, \beta}=k_{\beta, \alpha},
$$


then (4.18) can be rewritten as:

$$
\widetilde{k}_{\beta, T-1}+\sum_{j=1}^{T-1} \widetilde{C}_{\beta, j}^{T} \widetilde{k}_{j, T-1}+\widetilde{C}_{\beta, T}^{T}=0, \quad \beta=1, \ldots, T-1 .
$$

The last equation is an analog of Gelfand-Levatan equation for continuous problem $[4,5]$. We conclude this section with

Theorem 4. The kernel of the operator $\left(\bar{W}^{T}\right)^{-1}$ (see (4.10)) satisfies equation (4.20), where the entries $\widetilde{C}_{i, j}^{T}$ are defined in (4.17), (3.6).

The equation in (4.18) has a unique solution due to the invertibility of $C^{T}$. The potential can be recovered by (4.16).

Now, we make some remarks on the dependence of the connecting operator $C^{T}$ and the solution of the inverse problem equations (i.e. the potential) on the inverse data. As a direct consequence of (3.6) we can formulate the following:

Remark 1. The operator $C^{T}$ depends on $R^{2 T-2}$, i.e. it depends on the potential $\left(b_{1}, \ldots, b_{T-1}\right)$, so the results obtained from $C^{T}$ via Krein-type equations (4.4), (4.6),(4.7), factorization method (4.15), (4.16) and GelfandLevitan type equations (4.20), (4.16) are the best possible.

In the subsection on the factorization method, we used the fact that $\operatorname{det} C^{\tau}=1, \tau=2, \ldots, T$. More precisely, we used it in the form $\operatorname{det}\left(\bar{c}_{1}^{\tau}, \bar{c}_{2}^{\tau}, \ldots \bar{c}_{\tau}^{\tau}\right)=1$. That fact actually says that not all elements in the response vector are independent. Indeed: the element $k_{11}$ we recovered (see (4.12)) from $\bar{c}_{21}$, i.e. from $r_{1}$. The element $k_{22}$ we recovered from $\bar{c}_{11}, \bar{c}_{13}, \bar{c}_{21}, \bar{c}_{23}$, that is from $r_{1}, r_{2}, r_{3}$. But since $\operatorname{det}\left(\bar{c}_{1}^{2}, \bar{c}_{2}^{2}\right)=1$, we have that $r_{2}=r_{1}^{2}$, so in fact $k_{22}$ was recovered from $r_{1}$ and $r_{3}$. Arguing in the same fashion, we see that $r_{2 k}$ depends on $r_{2 l+1}$, $l=0, \ldots, k-1$. So we recovered $\left(k_{11}, \ldots, k_{T-1, T-1}\right)$ from the response vector $\left(r_{0}, r_{1}, \ldots, r_{2 t-2}\right), r_{0}=1$, whose components with even numbers depend in explicit form on the components with odd numbers. That observation plays an important role in the next subsection.

\subsection{Characterization of the inverse data}

In the second section, we considered the forward problem $(1.1)$, for the potential $\left(b_{1}, \ldots, b_{T-1}\right)$ we constructed the matrix $W^{T}$ (2.1), (2.2), the response vector $\left(1, r_{1}, \ldots, r_{2 T-2}\right)$ (see (3.1)) and the connecting operator $C^{T}$ by formula (3.6). It will be more convenient for us to deal with the rotated matrix $\bar{C}^{T}$ defined in (4.9). From the representation $\bar{C}^{T}=\left(\bar{W}^{T}\right)^{*} \bar{W}^{T}$ and triangularity of $\bar{W}^{T}$ we know that

$$
\operatorname{det} \bar{C}^{l}=1 \quad \forall l=1, \ldots, T \text {. }
$$

Also, we have proven that if coefficients $r_{1}, \ldots, r_{2 T-2}$ correspond to some potential $\left(b_{1}, \ldots, b_{T-1}\right)$, then we can recover the potential using (4.15)-(4.16).

Now, we set up a question: can one determine whether a vector $\left(1, r_{1}, r_{2}, \ldots, r_{2 T-2}\right)$ is a response vector for the dynamical system (1.1) with a potential $\left(b_{1}, \ldots, b_{T-1}\right)$ or not? The answer is the following theorem.

Theorem 5. The vector $\left(1, r_{1}, r_{2}, \ldots, r_{2 T-2}\right)$ is a response vector for the dynamical system (1.1) if and only if the matrix $C^{T}$ (3.6) is positively definite and $\operatorname{det} C^{l}=1, l=1, \ldots, T$.

Proof. First we observe that in the conditions of the theorem we can substitute $C^{T}$ by $\bar{C}^{T}$ (4.9). The necessary part of the theorem is proved in the preceding sections. We are left to prove the sufficiency of these conditions.

Let there be a vector $\left(1, r_{1}, \ldots, r_{2 T-2}\right)$ such that the matrix $\bar{C}^{T}$ constructed from it using (4.9) satisfies conditions of the theorem. Then we can construct the potential $\left(b_{1}, \ldots, b_{T-1}\right)$ using (4.15)-(4.16) and consider the dynamical system (1.1) with this potential. For this system, we construct the connecting operator $C_{n e w}^{T}$ and its rotated $\bar{C}_{\text {new }}^{T}$ using (2.2), (3.1), (3.6) and (4.9). We will show that the matrices $\bar{C}^{T}$ and $\bar{C}_{n e w}^{T}$ coincide.

First, we note that we have two matrices constructed by (4.9), one comes from the vector $\left(1, r_{1}, \ldots, r_{2 T-2}\right)$ and the other comes from $\left(1, r_{1}^{n e w}, \ldots, r_{2 T-2}^{n e w}\right)$. Also they have a common property that $\operatorname{det} \bar{C}^{l}=\operatorname{det} \bar{C}_{\text {new }}^{l}=1$ for all $l=1, \ldots, T$ (one by theorem's condition and the other by representation $\bar{C}_{\text {new }}^{T}=\left(\bar{W}_{\text {new }}^{T}\right)^{*} \bar{W}_{\text {new }}^{T}$ ).

Secondly, we note that if we calculate the potential $\left(b_{1}, \ldots, b_{T-1}\right)$ using (4.15)-(4.16) from any of $\bar{C}^{T}$ and $\bar{C}_{n e w}^{T}$ matrices, we obtain the same answer. 
Therefore we have two matrices of the type (4.9) with the unit principal minors and the property

$$
\operatorname{det}\left(\bar{c}_{1}^{l+1}, \ldots \bar{c}_{l}^{l+1}, \bar{c}_{l+2}^{l+1}\right)=\operatorname{det}\left({\overline{c_{\text {new }}}}_{1}^{l+1}, \ldots{\overline{c_{\text {new }}}}_{l}^{l+1},{\overline{c_{\text {new }}}}_{l+2}^{l+1}\right) \quad \forall l=0, \ldots, T-2 .
$$

If we look at (4.21) for $l=0$, we see that $r_{1}=r_{1}^{\text {new }}$. From the fact that for both matrices $\bar{C}^{T}, \bar{C}_{\text {new }}^{T}$ the principal minors of the second order are equal to one, we infer that $r_{2}=r_{2}^{\text {new }}$. We continue this procedure, and from (4.21) with $l=n$, we infer that $r_{2 n+1}=r_{2 n+1}^{n e w}$ and from equality to one of principal minor of the order $n+2$ of $\bar{C}^{T}$, $\bar{C}_{n e w}^{T}$, we can infer that $r_{2 n+2}=r_{2 n+2}^{n e w}$ for all $n=2, \ldots, T-2$ by induction. This finishes the proof.

\section{Spectral representation of $\mathrm{C}^{\mathrm{T}}$ and $\mathbf{r}_{t}$}

In this section, we consider the inverse spectral problem and show the connection of the spectral (2.5), (2.6) and dynamical (3.1), (3.2) inverse data. If we introduce the special control $\delta=(1,0,0, \ldots)$, then the kernel of response operator (3.2) is:

$$
r_{t}^{i}=\left(R_{i} \delta\right)_{t}=v_{1, t}^{\delta},
$$

on the other hand, we can use (2.9), (2.10) to obtain:

$$
v_{1, t}^{\delta}=\sum_{k=1}^{N} \frac{1}{\rho_{k}} T_{t}\left(\lambda_{k}\right) .
$$

So on introducing the spectral function:

$$
\rho^{N}(\lambda)=\sum_{\left\{k \mid \lambda_{k}<\lambda\right\}} \frac{1}{\rho_{k}}
$$

from (5.1), (5.2) we deduce that:

$$
r_{t}^{i}=\int_{-\infty}^{\infty} T_{t}(\lambda) d \rho^{N}(\lambda), \quad t \in \mathbb{N}
$$

Let us evaluate $\left(C_{i}^{T} f, g\right)$ for $f, g \in \mathcal{F}^{T}$, using the expansion (2.9):

$$
\begin{aligned}
\left(C_{i}^{T} f, g\right)=\sum_{n=1}^{N} v_{n, T}^{f} v_{n, T}^{g}= & \sum_{n=1}^{N} \sum_{k=1}^{N} \frac{1}{\rho_{k}} T_{T}\left(\lambda_{k}\right) * f \varphi_{n}^{k} \sum_{l=1}^{N} \frac{1}{\rho_{l}} T_{T}\left(\lambda_{l}\right) * g \varphi_{n}^{l} \\
& =\sum_{k=1}^{N} \frac{1}{\rho_{k}} T_{T}\left(\lambda_{k}\right) * f T_{T}\left(\lambda_{k}\right) * g=\int_{-\infty}^{\infty} \sum_{l=0}^{T-1} T_{T-l}(\lambda) f_{l} \sum_{m=0}^{T-1} T_{T-m}(\lambda) g_{m} d \rho^{N}(\lambda)
\end{aligned}
$$

From the equality above, it is evident that (cf. (3.6)):

$$
\left\{C_{i}^{T}\right\}_{l+1, m+1}=\int_{-\infty}^{\infty} T_{T-l}(\lambda) T_{T-m}(\lambda) d \rho^{N}(\lambda), \quad l, m=0, \ldots, T-1 .
$$

Let us consider the spectral problem:

$$
\left\{\begin{array}{l}
\phi_{i+1}+\phi_{i-1}-b_{n} \phi_{i}=\lambda \phi_{i}, \quad n=0, \ldots, N+1, \\
\phi_{0}=0, \phi_{N+1}=0 .
\end{array}\right.
$$

In the second section, we construct the spectral data for this problem - eigenvalues of the corresponding Hamiltonian and norming coefficients (2.5), (2.6). Now we answer the question how to recover the potential $\left(b_{1}, \ldots, b_{N}\right)$ from this data.

Our strategy will be to use the dynamical approach from the fourth section to treat this IP. First, we observe that to know (2.6) is the same as to know the spectral function (5.3). Consider the system (1.1) with the same potential $b_{n}$ for $n=1, \ldots, N$. We notice that as explained in the beginning of section four, $R^{2 N}=R_{i}^{2 N}$ and correspondingly, $r_{t}=r_{t}^{i}, t=1, \ldots, 2 N$. Due to this, we deduce that $C^{T}=C_{i}^{T}$ for $T=N+1$. Thus, the inverse 
problem can be solved in the following way: from the spectral data (2.6), we construct the spectral function by (5.3). Then, we construct:

$$
\begin{gathered}
r_{t}=r_{t}^{i}=\int_{-\infty}^{\infty} T_{t}(\lambda) d \rho^{N}(\lambda), \quad t=1, \ldots, 2 N \\
C_{l m}^{T}=\left\{C_{i}^{T}\right\}_{l+1, m+1}=\int_{-\infty}^{\infty} T_{T-l}(\lambda) T_{T-m}(\lambda) d \rho^{N}(\lambda), \quad l, m=0, \ldots, N-1 .
\end{gathered}
$$

After we have in hands the connecting operator, we can use the methods of section four to find $\left(b_{1}, \ldots, b_{N}\right)$.

\section{Acknowledgements}

The research of Victor Mikhaylov was supported in part by NIR SPbGU 11.38.263.2014 and RFBR 14-0100535. Alexandr Mikhaylov was supported by RFBR 14-01-00306; A. S. Mikhaylov and V.S. Mikhaylov were partly supported by VW Foundation program 'Modeling, Analysis, and Approximation Theory toward application in tomography and inverse problems'.

\section{References}

[1] Belishev M.I. Recent progress in the boundary control method. Inverse Problems, 2007, 23 (5), R1.

[2] Avdonin S.A., Mikhaylov A.S., Mikhaylov V.S. On some applications of the Boundary Control method to spectral estimation and inverse problems. Nanosystems: Phys. Chem. Math., 2015, 6 (1), P. 63-78.

[3] Belishev M.I. C*-Algebras in reconstruction of manifolds. Nanosystems: Phys. Chem. Math., 2013,4 (4), P. $484-489$.

[4] Avdonin S.A., Mikhaylov V.S. The boundary control approach to inverse spectral theory. Inverse Problems, 2010,26 (4), 045009,19 p.

[5] Belishev M.I., Mikhailov V.S. Unified approach to classical equations of inverse problem theory. Journal of Inverse and Ill-Posed Problems, 2012, 20 (4), P. 461-488. 